\title{
Qualitätsarbeit muss sich am Nutzen orientieren
}

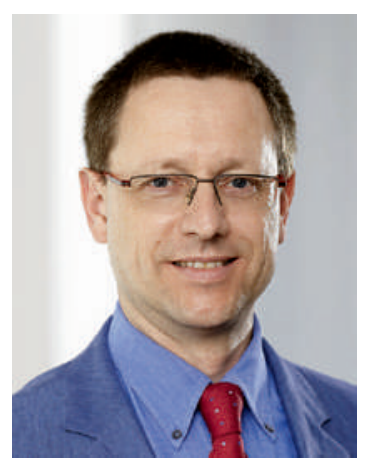

Die Qualität sei eine Hure, hielt mir ein ansonsten stets für seine Ruhe und Gelassenheit bekannter Kollege entgegen, als ich mit ihm neulich diskutierte. Er war genervt über für ihn unklare Begrifflichkeiten. Irritiert ging ich nach Hause - als QualitätsVerantwortlicher der FMH hätte ich eigentlich ad hoc mit einer zutreffenden Definition kontern müssen.

Immer wenn es schwierig wird, frage ich meine Kinder um Rat. Und sie hatten sofort eine griffige Definition zur Hand: Qualität ist, wenn ich erhalte, was ich will. Qualität ist somit kein absoluter Begriff, sondern stets relativ zu sehen. Je nach Interessen, Bedürfnissen, und Aufgaben sind andere Qualitäten gefragt. Ebenso werden unterschiedliche Grössenordnungen und Priorisierungen angestrebt.

Diese Pluralität der Sichtweisen ist letztlich bereichernd, um aus einer allzu eindimensionalen Position herauszufinden. Die Qualitätsthematik macht uns aber auch immer wieder bewusst, dass wir mit Begrifflichkeiten und Vorgehensweisen an Grenzen stossen: Was für den einen gut ist,

\section{Qualität ist keine absolute Grösse, sondern definiert sich nach Absichten und Zielen unterschiedlich.}

muss nicht für alle gut sein; was in einer besonderen Situation gut ist, muss nicht immer und überall gut sein. Mit Verlaub: Ich benutze den Brat-Thermometer auch nicht zur Messung der Schnee-Temperatur zwecks Wahl des Ski-Wachses.

Oft bedienen sich Verwaltung und Politik im Gesundheitswesen des Begriffs Qualität. Im KVG werden die Leistungserbringer dazu aufgefordert, Qualitäts-Indikatoren zur
Verfügung zu stellen. Nun ist aber die Sichtweise des Staates, welcher per Verfassung die Versorgung garantieren muss, eine andere als jene der Versicherer, welche die Finanzen bereitstellen sollen. Und ebenso hat die Ärzteschaft nochmals einen anderen Blickwinkel, welche gemeinsam mit ihren Patienten ein individuell formuliertes Ziel im Kontext der aktuellen gesundheitlichen Problematik erreichen will.

\section{Projekte, die den Patienten dienen und das Qualitätsmanagement im Gesund- heitswesen voranbringen, stehen beim Swiss Quality Award im Mittelpunkt.}

Trotzdem - auch in der Qualitätsthematik geht es vorwärts: Dies beweisen die Gewinnerinnen und Gewinner des diesjährigen Swiss Quality Awards, welcher gemeinsam vom Institut für Evaluative Forschung in der Medizin IEFM der Universität Bern, der Schweizerischen Gesellschaft für Qualitätsmanagement im Gesundheitswesen SQMH sowie der FMH getragen wird. Erstmals wurde der Swiss Quality Award in den Preiskategorien Ambulanter Sektor, Stationärer Sektor und sektorübergreifend verliehen. Und ebenso vergab die Jury anlässlich des Symposiums neu einen Poster-Award.

Die Gewinnerprojekte zeigen eindrücklich, dass mit klar formulierten Zielen, transparenten Grundlagen und Prozessen die Qualitätsarbeit mit Erfolg belohnt wird - und dies immer zum Nutzen der Patientinnen und Patienten. Ich gratuliere den drei Gewinnerteams und bedanke mich im Namen der Trägerorganisationen auch für die weiteren spannenden Projekte, welche für den diesjährigen Swiss Quality Award eingereicht wurden.

Dr. med. Christoph Bosshard, Mitglied des Zentralvorstandes der FMH, Departementsverantwortlicher Daten, Demographie und Qualität / Schweizerische Akademie für Qualität in der Medizin SAQM 